\section{'Rubygem' Strawberry}

\author{
Mark E. Herrington ${ }^{1}$ \\ Department of Primary Industries and Fisheries, Maroochy Research \\ Station, P.O. Box 5083, Nambour, Queensland, 4560, Australia
}

Craig K. Chandler

University of Florida, Gulf Coast Research and Education Center, 14625

C.R. 672, Wimauma, FL 33598

Jennifer A. Moisander

Department of Primary Industries and Fisheries, Redlands Research Station, P.O. Box 327, Cleveland, Queensland, 4163, Australia

\section{Claire E. Reid}

Department of Primary Industries and Fisheries, Innovative Food Technologies, 19 Hercules Street, Hamilton, Queensland, 4007, Australia

Additional index words. Fragaria $\times$ ananassa, fruit breeding

\begin{abstract}
Rubygem', a new short-day strawberry (Fragaria $\times$ ananassa Duch.), produces high yields of moderately firm, attractive well-flavored fruit from late autumn through early spring in the strawberry-growing district in Southeast Queensland. 'Rubygem' is recommended for trial in areas with mild winter climates, especially where rainfall is unlikely and a well-flavored berry is required.
\end{abstract}

There is a need in Queensland winter strawberry (Fragaria $\times$ ananassa Duch.) production areas for a highly flavored, earlyripening cultivar to replace or be an alternative to 'Kabarla' (Herrington, 1995). 'Kabarla' has benefited the Queensland strawberry industry through its high production of fruit early in the season, when market prices are typically high. However the shape of 'Kabarla' fruit is often irregular, with a high frequency of small fruit, which limit its marketability when fruit of other cultivars are readily available. 'Sweet Charlie' (Chandler et al., 1997) and 'Earlibrite' (Chandler et al., 2000a) have been tested in Queensland as early-yielding cultivars, but have not become established because they lack sufficient earliness, resistance to bruising, or uniformity of fruit shape. 'Strawberry Festival' (Chandler et al., 2000b) was recently introduced to Queensland and has rapidly fruit and plant attributes desirable to growers, although 'Strawberry Festival' commences production slightly after 'Kabarla'. The cultivar 'Rubygem' was developed to provide early-season production of high-flavored fruit. 'Rubygem' strawberry has produced high early-season (May through August)

Received for publication 27 Jan. 2007. Accepted for publication 29 Apr. 2007. Australia Limited under project no. BS01005, Department of Primary Industries and Fisheries, and Strawberries Australia.

We thank Louella Woolcock, Department of Primary Industries and Fisheries, for her technical support.

${ }^{1}$ To whom reprint requests should be addressed; e-mail mark.herrington@dpi.qld.gov.au become a major early-season cultivar with

Funding support was provided by Horticulture librite', a 2000 release from the Florida Agricultural Expt. Sta., was used as a parent because of its high early-season yield potential and its desirable shape and low acidity. 'Carlsbad', a 1994 release from the University of California, was used as a parent because of its ability to produce firm, attractive fruit. 'Rubygem' was first selected as a desirable genotype based on appearance, flavor, resistance to bruising, and suitable plant characteristics in Queensland during the 1999 season, and was identified as 'Selection 99-194' before being released as 'Rubygem'.

\section{Description}

'Rubygem' is a short-day cultivar. It has a more compact but upright plant habit than 'Kabarla' and is similar to 'Strawberry Festival'. The leaf color of the upper side is medium green (137B) (Royal Horticultural Society, 1995), with a slightly concave shape in cross-section. Blistering is absent or very weak and glossiness is weak. The terminal leaflet's length-to-width ratio is longer than broad (average, 1.08), with an obtuse shape of the leaf base. Incisions of leaf margins are crenate, and the attitude of hairs on the petiole is strongly outward. Stipule anthocyanin coloration is absent or very weak. The compact plant habit and relatively long and stiff pedicels result in many flowers and fruit that are exposed. This exposure makes the fruit easy to harvest, but also increases its vulnerability to rain damage. 'Rubygem' produces fruit that are similar in weight to 'Strawberry Festival' (Table 1). Fruit are mostly conical to cordiform, with some short wedge in shape (Fig. 1). However, fruit are not always as regularly shaped as those of 'Strawberry Festival'. External fruit color is red (44A) and glossy, and the flesh is a medium red (43A) (Royal Horticultural Society, 1995). The calyx is much larger than the fruit diameter, especially on the primary fruit of flushes. Fruit of 'Rubygem' are firm yet juicy, and the flavor and overall acceptability of the fruit are as highly regarded as that of 'Camarosa' (Table 2). and 'Carlsbad' (Voth et al., 1994). 'Ear-

Table 1. Cumulative yield, fruit weight, soluble solids, and titratable acidity of 'Rubygem' in comparison with selected standard cultivars grown in the field at Cleveland, Queensland, during the 2005 season.

\begin{tabular}{|c|c|c|c|c|c|c|c|}
\hline \multirow[b]{2}{*}{ Cultivar $^{z}$} & \multicolumn{3}{|c|}{ Cumulative yield (g/plant) ${ }^{y}$} & \multirow[b]{2}{*}{$\mathrm{Wt} /$ fruit $(\mathrm{g})^{\mathrm{x}}$} & \multirow{2}{*}{$\frac{\text { Soluble solids }(\%)^{\mathrm{w}}}{2005}$} & \multicolumn{2}{|c|}{ Titratable acidity (\%) } \\
\hline & June & July & August & & & 2003 & 2005 \\
\hline Rubygem & $152 \mathrm{ab}$ & $359 \mathrm{a}$ & $700 \mathrm{a}$ & $22.8 \mathrm{~b}$ & $9.1 \mathrm{ab}$ & $0.51 \mathrm{ab}$ & $0.48 \mathrm{c}$ \\
\hline Festival & $150 \mathrm{ab}$ & $368 \mathrm{a}$ & $610 \mathrm{a}$ & $23.3 \mathrm{~b}$ & $9.4 \mathrm{ab}$ & $0.55 \mathrm{bc}$ & $0.51 \mathrm{c}$ \\
\hline Kabarla & $153 \mathrm{a}$ & $393 \mathrm{a}$ & $660 \mathrm{a}$ & $19.3 \mathrm{c}$ & $9.9 \mathrm{a}$ & $0.79 \mathrm{e}$ & $0.77 \mathrm{a}$ \\
\hline Camarosa & $25 \mathrm{~d}$ & $236 \mathrm{~b}$ & $433 \mathrm{~b}$ & $26.0 \mathrm{a}$ & $8.6 \mathrm{~b}$ & $0.63 \mathrm{~cd}$ & $0.74 \mathrm{ab}$ \\
\hline Selva & $122 \mathrm{~b}$ & $232 \mathrm{~b}$ & $347 \mathrm{~b}$ & $18.6 \mathrm{c}$ & $9.0 \mathrm{ab}$ & $0.64 \mathrm{~d}$ & $0.68 \mathrm{~b}$ \\
\hline
\end{tabular}

${ }^{2}$ Commercial 'Rubygem', 'Festival', and 'Kabarla' planted "leaf on" 31 Mar.; 'Selva' and 'Camarosa' planted "leaf off" 7 and 12 Apr. 2005 for yield and fruit size data. Leaf-on transplants have intact leaves whereas leaf-off transplants have the upper half of the petiole and all leaf blades removed.

${ }^{\mathrm{y}}$ Within columns, values followed by a letter in common are nonsignificant according to analysis of variance with LSD $(P \leq 0.05$; GenStat Eighth Edition). Means are of three replications each of 15 plants with 40-cm intrarow and 35-cm interrow on double-row, black polythene, mulched beds at 155 -cm centers. In Queensland June is the first month of winter.

${ }^{\mathrm{x}}$ Mean fruit weight was determined by dividing total marketable fruit yield per plot by total marketable fruit number per plot.

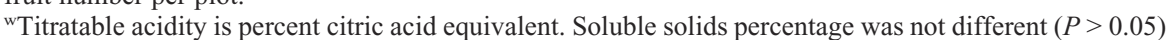
among cultivars in 2003 (7.9\%; data not shown). Assessed 15 July 2003 and 28 July 2005. 


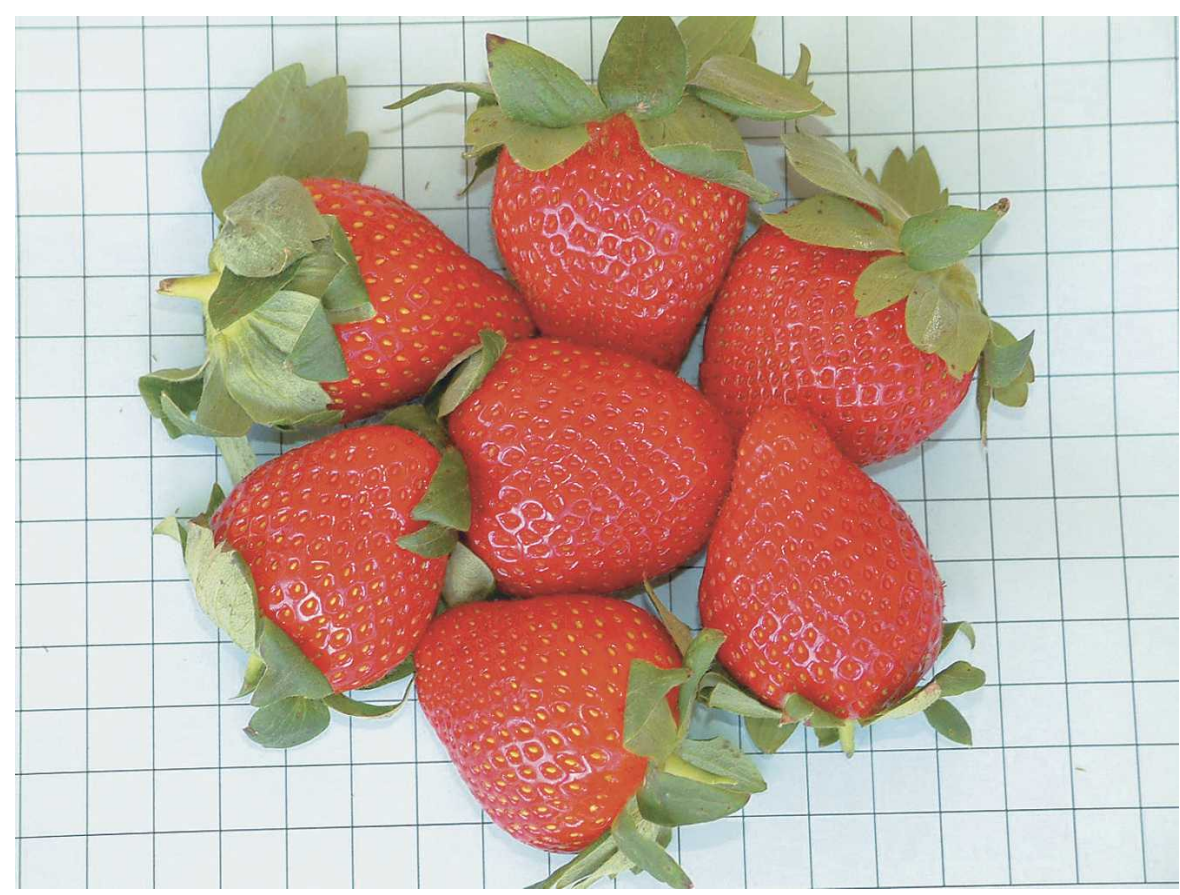

Fig. 1. Fruit of 'Rubygem' strawberry. Grid, 1-cm squares.

Table 2. Sensory characteristics of overall, flavor and texture "acceptability," and juiciness, sweetness, and acidity "just right" of 'Rubygem' in comparison with selected standard cultivars in 2005.

\begin{tabular}{lcccccc}
\hline Cultivar & Overall & Flavor & Texture & Juiciness & Sweetness & Acidity \\
\hline Rubygem & $69 \mathrm{a}$ & $64 \mathrm{a}$ & $69 \mathrm{a}$ & $51 \mathrm{a}$ & $40 \mathrm{a}$ & $53 \mathrm{~b}$ \\
Festival & $63 \mathrm{a}$ & $57 \mathrm{ab}$ & $63 \mathrm{~b}$ & $47 \mathrm{~b}$ & $35 \mathrm{~b}$ & $55 \mathrm{ab}$ \\
Kabarla & $56 \mathrm{~b}$ & $52 \mathrm{~b}$ & $59 \mathrm{~b}$ & $41 \mathrm{c}$ & $33 \mathrm{~b}$ & $60 \mathrm{a}$ \\
Camarosa & $64 \mathrm{a}$ & $61 \mathrm{a}$ & $64 \mathrm{ab}$ & $52 \mathrm{a}$ & $42 \mathrm{a}$ & $56 \mathrm{ab}$ \\
\hline
\end{tabular}

Predicted means from the analysis of the average ratings of 21 to 24 untrained panelists. Data were analyzed using residual maximum likelihood with cultivars as a fixed effect, and blocks and sessions within blocks as random effects. Mean separation within columns by Fisher's protected LSD $(P \leq 0.05)$. Acceptability of overall flavor, appearance, and texture scored on a hedonic line scale from dislike extremely ( 0 point), neither like nor dislike (50 points), to like extremely (100 points); "just right" level of juiciness, firmness, sweetness, and acidity scored on a scale from not enough characteristic ( 0 point), just right (50 points), through too much of characteristic (100 points). There was no difference among cultivars for firmness (50 points) or appearance ( 70 points; data not shown).

\section{Performance}

'Rubygem' was included in replicated variety trials at Maroochy Research Sta., Nambour, during 2002 through 2005, and Redlands Research Sta., Cleveland, in 2005. Ripe fruit were harvested, graded, counted, and weighed weekly from May through August. Acidity, as percent citric acid equivalents, and soluble solids ( ${ }^{\circ}$ Brix) were assessed in 2003 and 2005. For postharvest taste panel analysis, ripe fruit were harvested at Redlands Research Sta. 23-25 Aug. 2005 and transported the same day to the sensory evaluation facilities at DPI\&F Hamilton, Queensland. Twenty one to 24 untrained panelists were asked to rate a sample of the fruit according to an incomplete block design higher ( $\mathrm{LSD}_{0.05}, 60 \mathrm{~g} / \mathrm{plant}$ ) early yields to the end of June than 'Strawberry Festival' (100 $\mathrm{g} /$ plant) and was similar to Kabarla (132 g/plant). 'Rubygem' has been shown to have resistance to fusarium wilt (Fusarium oxysporum Schlecht. Ex Fr. f. sp. fragariae, Winks and Williams) at a similar level to 'Strawberry Festival' and more resistant than that of 'Selva' (Hutton and Gomez, 2006). Although the relative susceptibility to anthracnose fruit rot (Colletotrichum acutatum Simmonds), colletotrichum crown rot (Colletotrichum gloeosporioides Penz.), botrytis fruit rot (Botrytis cinerea Pers. ex Fr.), powdery mildew [Sphaerotheca macularis (Wallr. ex Fr.) Jacz. f. sp. fragariae], or to the two-spotted spider mite (Tetranychus urticae Koch) has not been determined, serious epidemics of these diseases and infestations of this pest have not been observed when appropriate control measures (such as clean planting material, standard fungicide applications, and predatory mite releases) have been used. However, during rain events, fruit of 'Rubygem' are more susceptible to cracking (55\%) than those of 'Strawberry Festival' (30\%).

\section{Availability}

'Rubygem' has been officially registered by DPI\&F and Horticulture Australia Limited as 'DPI Rubygem'. Plants or licenses for 'Rubygem' can be accessed through Ekland Marketing Company, P.O. Box 6067, Chico, CA, 95927, and through Garry Fullelove, DPI\&F Forestry Science Building, 80 Meiers Road, Indooroopilly, Queensland, 4168 Australia, Garry.Fullelove@dpi.qld.gov.au.

\section{Literature Cited}

Chandler, C.K., E.E. Albregts, C.M. Howard, and J.K. Brecht. 1997. 'Sweet Charlie' strawberry. HortScience 32:1132-1133.

Chandler, C.K., D.E. Legard, D.D. Dunigan, T.E. Crocker, and C.A. Sims. 2000a. 'Earlibrite' strawberry. HortScience 35:1363-1365.

Chandler, C.K., D.E. Legard, D.D. Dunigan, T.E Crocker, and C.A. Sims. 2000b. 'Strawberry Festival' strawberry. HortScience 35:13661367

Herrington, M. 1995. 'Kabarla'. Plant Varieties J. 8:50-51.

Hutton, D. and A. Gomez. 2006. The incidence of fusarium wilt in Queensland cultivars, p. 193198. In: C.M. Menzel and G.K. Waite (eds.). Horticulture Australia projects BS01002, BS04001 and BS05003. Horticulture Australia Ltd., Sydney.

Royal Horticultural Society. 1995. Royal Horticultural Society colour chart. Royal Horticultural Society, London, UK

Voth, V., D.V. Shaw, and R.S. Bringhurst. 1994. Strawberry plant called 'Carlsbad'. United States plant patent no. 8660 . 